\title{
Metodologias para quantificação do fator de empilhamento em vegetação de caatinga/cerrado
}

\author{
Lucas de Souza Silva ${ }^{1 *}$, Huga Géssica Bento de Oliveira ${ }^{1}$, Mylla Vyctória Coutinho Sousa ${ }^{1}$, Lailla \\ Sabrina Queiroz Nazareno', Andressa Ribeiro'1, Antônio Carlos Ferraz Filho' ${ }^{1}$
}

\begin{abstract}
RESUMO: Objetivou-se testar metodologias alternativas para caracterizar o volume sólido das pilhas de madeira bem como descrever o fator de empilhamento $(\mathrm{Fe})$ para uma vegetação transicional de caatinga/cerrado. $\mathrm{O}$ estudo foi realizado em uma área experimental Fazenda Aracajú, Cristino Castro, Piauí. Os dados foram coletados em 11 pilhas de madeiras dispostas nas bordas de uma área com 30 por 90 metros. O Fe foi calculado pela razão entre o volume empilhado pelo volume sólido. Para testar a redução da amostragem, utilizou-se 7 tratamentos, sendo: T1: face A e B e um diâmetro (horizontal); T2: face A e B e um diâmetro (vertical); T3: face A e diâmetros cruzados; T4: face B e diâmetros cruzados; T5: face A e um diâmetro; T6: face B e um diâmetro. As análises foram feitas em planilha eletrônica e o teste t pareado (95\% de probabilidade de acerto) foi adotado para comparação dos tratamentos. O volume sólido médio foi de $1,57 \mathrm{~m}^{3}$ e o volume empilhado médio foi de 4,03 mst. O Fe foi de 2,55. O tratamento T6 obteve bom resultado, demonstrando a possibilidade da redução de amostragem medindo apenas uma face e um diâmetro nas pilhas.
\end{abstract}

Palavras-chave: volume sólido, volume empilhado, pilhas de madeira.

\section{Methodologies for obtaining solid to stacked wood conversion factor for caatinga/cerrado}

\begin{abstract}
Alternative methodologies were used to characterize the solid volume of wood piles as well as to describe the conversion factor from solid to stacked wood (Fe) for a transitional caatinga/cerrado vegetation. The study was carried out in an experimental area of Fazenda Aracajú, Cristino Castro, Piauí. The data were collected in 11 stacks of wood arranged on the edges of an area with 30 by 90 meters. The Fe was calculated by the reasoning of Stacked Volume by Solid Volume. To test the reduction of the sample, 7 treatments: T1: face A and B of the wood pile and one diameter (horizontal); T2: face $A$ and B and one diameter (vertical); T3: face A and cross-diameters; T4: face $B$ and cross diameters; T5: face $A$ and one diameter; T6: face B and one diameter. The analyzes were done in a spreadsheet using the paired t-test (95\% probability of success) was adopted to compare the treatments. The mean solid volume was $1.57 \mathrm{~m}^{3}$ and the mean stacked volume was $4.03 \mathrm{mst}$. The Fe from solid to stacked wood was 2.55 . Treatment T6 obtained good results, demonstrating the possibility of reducing sampling by measuring only one face and one diameter.
\end{abstract}

Keywords: solid volume, stacked volume, stacked wood.

\section{INTRODUÇÃO}

O consumo de lenha é relevante no Brasil para produção energética. Segundo o Balanço Energético Nacional das 74.500 mil toneladas de lenha produzidas no Brasil, 51.602 mil foram destinadas ao consumo energético, cerca de $69,26 \%$ da produção total (BRASIL, 2017).

As vantagens do uso da madeira consistem na redução de custos e impactos ao meio ambiente, se comparada aos combustíveis derivados do petróleo (NASCIMENTO, BIAGGIONI, 2010). Segundo Brito (2007) o uso da madeira possui alto potencial renovável e produtivo e sua matriz energética ambientalmente saudável e socialmente justa possibilita maiores taxas de geração de emprego por recurso monetário investido.

Contudo, a exploração da madeira deve ser realizada de maneira responsável, adotando técnicas de Manejo Florestal Sustentável, com intuito de garantir o abastecimento contínuo do mercado consumidor com matéria prima energética (SANTOS et al., 2017). Uma atividade imprescindível para o Plano de Manejo Florestal Sustentável é a mensuração do volume de madeira, principalmente para a comercialização das toras.

Em termos de praticidade, o volume das toras para comercialização é mensurado em pilhas. $\mathrm{O}$ volume de madeira empilhada pode ser obtido através da mensuração das dimensões da pilha, que produz um volume em metro estéreo (mst), que por sua vez pode ser convertido em volume sólido $\left(\mathrm{m}^{3}\right)$ aplicando o fator de empilhamento (MACHADO, FIGEIREDO FILHO, 2009).

O volume empilhado detém da praticidade na medição em campo, já o volume sólido é aquele que

\footnotetext{
Recebido em 05/10/2019, Aceito para publicação em 15/10/2019

${ }^{1}$ Universidade Federal do Piauí

*e-mail: lucas045furtado@gmail.com
} 
representa o que é efetivamente comercializável, e por isso, caracteriza a produção de madeira (BATISTA, COUTO, 2002). No entanto, este requer grande esforço, tempo e mão-de-obra para sua caracterização (SOARES et al., 2003).

Diante disso, o presente trabalho objetivou testar metodologias alternativas para caracterizar o volume sólido das pilhas de madeira utilizando um fator de empilhamento, determinado sob diferentes óticas de medição, para uma vegetação transicional de caatinga/cerrado.

\section{MATERIAL E MÉTODOS Caracterização da área}

O estudo foi realizado com dados coletados na Fazenda Aracajú, situada no município de Cristino Castro, no estado do Piauí, entre as coordenadas

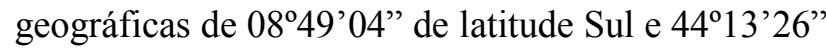
de longitude Oeste, com área total de 5.248,80 mil hectares.

A fazenda apresenta vegetação arbórea característica de caatinga hipoxerófila e elementos de cerrado, o que pode classificá-la como uma área de transição. Desta vegetação é realizada extração de madeira para fins energéticos sob um Plano de Manejo Florestal Sustentável (PMFS) devidamente regulamentado pelo IBAMA, sendo a empresa Gurguéia Manejo Florestal Ltda. a responsável pela exploração.

O clima do local segundo classificação de Koppen é Aw (tropical quente e úmido), apresentando inverno seco, verão chuvoso com preciptação média anual de $984,8 \mathrm{~mm}$ e temperatura média anual de $27^{\circ} \mathrm{C}$ com umidade média relativa do ar de 58,5\% (ALVARES et al., 2013).

\section{Coleta e Processamento dos dados}

Os dados foram coletados em 11 pilhas de madeiras cortadas e dispostas nas bordas de uma área com 30 por 90 metros (Figura 1). Foram utilizadas suta, fita métrica e trena para a aquisição das dimensões da pilha: comprimento total, altura e largura a cada 0,25 $\mathrm{m}$ de distância e, diâmetro cruzado de cada tora de ambas as faces. Posteriormente os dados foram tabulados e processados no programa Microsoft Excel®.

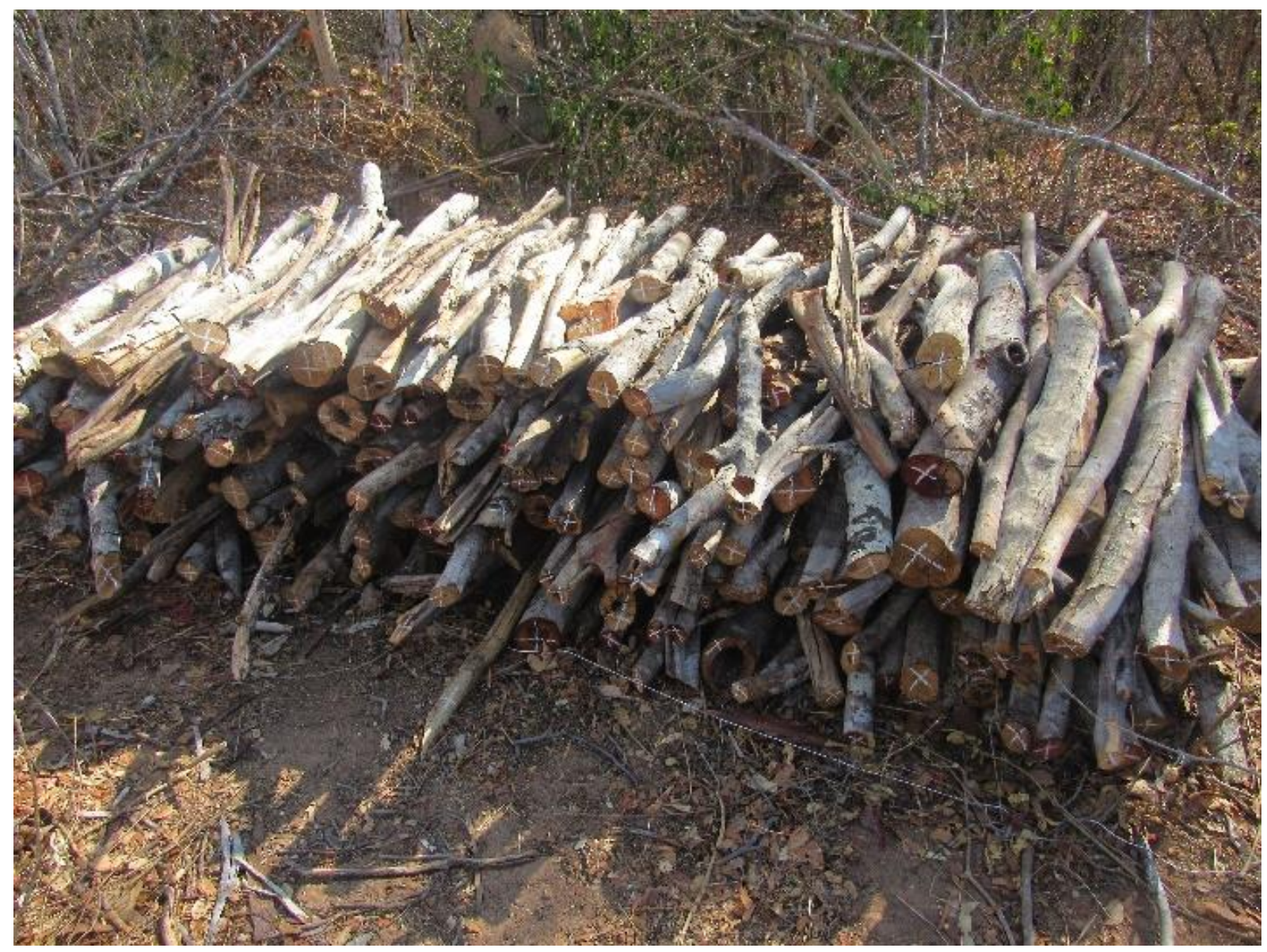

Figura 1 - Demonstração de uma das pilhas mensuradas.

No processamento dos dados foi seguida a metodologia apresentada em Machado, Figueiredo Filho (2009). O fator de empilhamento (Fe) foi calculado pela razão entre o volume empilhado e o volume sólido (Equação 1). A obtenção deste fator permite converter $1 \mathrm{~m}^{3}$ de madeira sólida para $1 \mathrm{mst}$ (metro estéreo) de madeira empilhada. 


$$
\left.F_{e}=\frac{\text { Volume empilhado }}{\text { Volume sólido }} \geq 1 \quad \text { (Equação } 1\right)
$$

O volume empilhado foi calculado obtendo a média da largura e altura da pilha a cada $0,25 \mathrm{~m}$ de distância e multiplicando-as pelo comprimento total da mesma (Equação 2). Este volume é dado em metro estéreo (mst) que caracteriza o volume das toras de madeira considerando os espaços vazios existentes na pilha decorrentes da forma como esta foi organizada e/ou das irreguralidades das toras presentes, como tortuosidade, forma, etc.

$$
\mathrm{V}_{\mathrm{e}}=\bar{h} * C * \bar{l} \quad(\text { Equação } 2)
$$

em que: $\mathrm{V}_{\mathrm{e}}$ : volume empilhado (mst); $\bar{h}$ : altura média a cada $0,25 \mathrm{~m} ; C$ : comprimento total; $\bar{l}$ : largura média a cada $0,25 \mathrm{~m}$.

O volume sólido é dado em metro cúbico $\left(\mathrm{m}^{3}\right)$ e não considera os espaços vazios presentes na pilha. Este volume foi calculado obtendo a somatória das áreas seccionais das toras de madeira de cada face, realizando a média das somatórias e multiplicando-a pela largura média da pilha (Equação 3).

$$
\mathrm{V}_{\mathrm{s}}=\frac{\left(\Sigma \mathrm{g}_{1}+\Sigma \mathrm{g}_{2}\right)}{2} * \bar{l} \quad(\text { Equação } 3)
$$

Tabela 1. Volume ( $\mathrm{m}^{3}$ e mst) e fator de empilhamento por pilha, referentes ao tratamento controle (T0).

\begin{tabular}{cccc}
\hline Pilha & Volume $\left(\mathbf{m}^{\mathbf{3}}\right)$ & Volume $(\mathbf{m s t})$ \\
\hline $\mathbf{1}$ & 1,11 & 3,19 & Fe \\
$\mathbf{2}$ & 1,59 & 4,22 & 2,88 \\
$\mathbf{4}$ & 1,75 & 4,64 & 2,65 \\
$\mathbf{5}$ & 1,47 & 4,09 & 2,79 \\
$\mathbf{6}$ & 1,58 & 4,14 & 2,62 \\
$\mathbf{7}$ & 1,25 & 2,96 & 2,36 \\
$\mathbf{8}$ & 1,59 & 3,80 & 2,38 \\
$\mathbf{9}$ & 1,42 & 3,32 & 2,34 \\
$\mathbf{1 0}$ & 2,30 & 6,02 & 2,62 \\
$\mathbf{1 1}$ & 1,23 & 2,62 & 2,13 \\
& 1,99 & 5,27 & 2,65 \\
\hline
\end{tabular}

Fonte: os autores.

Conforme observado na Tabela 1, a pilha 9 apresentou maior volume em métro cúbico, bem como em metro estéreo. Isto se deve ao fato das dimensões desta pilha serem maior que as demais, sendo 5,50 de comprimento, 0,94 de altura média e 1,17 de largura média.

A pilha 1 apresentou maior fator de empilhamento com o valor de 2,88 . Isso se deve à presença de toras de madeira de área seccional pequenas, sendo o somatório das áreas seccionais dos toretes desta pilha o menor dentre os demais. Essa afirmação é feita em cima do exposto por Campos, Leite (2013) citando o diâmetro e

em que: $V_{S}$ : volume sólido $\left(\mathrm{m}^{3}\right) ; \boldsymbol{\Sigma} \mathrm{g}_{1}$ : somatório da área seccional da face $\mathrm{A}$ da pilha; $\boldsymbol{\Sigma} \mathrm{g}_{2}$ : somatório da face B da pilha; $\bar{l}$ : largura média da pilha a cada $0,25 \mathrm{~m}$.

Foram testadas 4 metodologias de medição, sendo: I) mensuração dos diâmetros cruzados de todas as toras em ambas as faces da pilha (face A e face B); II) aferição de ambas as faces da pilha e um diâmetro de todas as toras; III) medição de uma face da pilha e diâmetros cruzados de todas as toras da mesma e IV) mensuração de uma face da pilha e apenas um diâmetro de todas as toras desta face.

As 4 metodologias estudadas resultaram em 7 tratamentos diferentes, sendo: T0: duas faces e diâmetros cruzados - tratamento controle; T1: face A e B e um diâmetro (horizontal); T2: face A e B e um diâmetro (vertical); T3: face A e diâmetros cruzados; T4: face B e diâmetros cruzados; T5: face A e um diâmetro; T6: face $\mathrm{B}$ e um diâmetro. A diferença entre os tratamentos foi avaliada utilizando o teste $\mathrm{t}$ pareado a 5\% de probabilidade por meio da planilha eletrônica do programa Microsoft Excel®.

\section{RESULTADOS E DISCUSSÃO}

Na Tabela 1 são apresentados as estimativas para o volume sólido de madeira $\left(\mathrm{m}^{3}\right)$, volume empilhado (mst) e fator de empilhamento de cada pilha mensurada, relativo ao tratamento controle (T0). 
energéticos no estado do Pernambuco, encontrou um fator de empilhamento de 4,70 para a algaroba.

A diferença do fator de empilhamento se deve ao número de espécies presentes na pilha, bem como as características morfológicas destas espécies (SCOLFORO et al., 2008). Conforme Campos, Leite (2013), a presença de espaços vazios nas pilhas de madeira interfere diretamente no fator de empilhamento, sendo esses espaços resultantes de fatores como diâmetro e comprimento das peças empilhadas, a forma como a pilha foi organizada, espécies ou material genético e madeira de copa ou de fuste.

$\mathrm{Na}$ Tabela 2 estão apresentados os valores do fator de empilhamento calculado para os diferentes tratamentos avaliados, bem como os resultados da análise estatística do teste t-pareado, com o valor de p.

Tabela 2. Resultados da análise estatística dos tratamentos analisados.

\begin{tabular}{cccccc}
\hline Tratamento & Fe & T-calc & Valor de p \\
\hline T0 & 2,55 & - & \\
T1 & 2,45 & 9,58 & \\
T2 & 2,58 & $-2,56$ & $0,00^{*}$ \\
T3 & 2,67 & $-2,35$ & $0,03 *$ \\
T4 & 2,46 & 2,21 & $0,04 *$ \\
T5 & 2,66 & $-2,41$ & 0,05 \\
T6 & 2,51 & 0,90 & $0,04 *$ \\
\hline
\end{tabular}

*= significativo ao nível de $5 \%$ de probabilidade.

Fonte: os autores.

Observa-se que com excessão do tratamento $\mathrm{T} 4 \mathrm{e}$ T6, todos os tratamentos obtiveram diferença significativa do tratamento controle (T0). O resultado do teste $\mathrm{t}$ demonstra um melhor desempenho do tratamento T6 se comparado ao T4. Sendo assim quando almeja-se redução do esforço e tempo para estimativa de volume sólido de pilhas, o tratamento T6 (apenas uma face e um diâmetro) é o mais indicado. O T5 apresentou diferença do T0, e em condições de campo não existe forma de escolher qual dos dois lados da pilha deve ser priorizado para a medição, recomenda-se a medição completa da pilha (T0) quando uma maior precisão é exigida para a quantificação do fator de empilhamento.

\section{CONCLUSÕES}

O fator de empilhamento médio foi de 2,55 para a área estudada. $\mathrm{O}$ fator de empilhamento dos tratamentos T4 e T6 não diferiram significativamente do tratamento controle. No entanto, o tratamento T6 apresentou melhor desempenho, mas quando uma maior precisão for exigida para a quantificação do fator de empilhamento, deve-se utilizar o T0.

\section{REFERÊNCIAS}

ALVARES, C. A.; STAPE, J. L.; SENTELHAS, P. C.; GONÇALVES, J. L. M.; SPAROVEK, G.; Köppen's climate classification map for Brazil. Meteorologische Zeitschrift, v. 22, n. 6, p. 711-728, 2013.

ARAÚJO, L. V. C.; PAULO, M. C. S.; PAES, J. B. Características dendrométricas e densidade básica da jurema-preta (Mimosa tenuiflora (Willd.) Poir) de duas regiões do Estado da Paraíba. Caatinga, Mossoró, v. 20, n. 1, p. 89-96, 2007.
BARROS, B. C. Volumetria, calorimetria e fixação de carbono em florestas plantadas com espécies exóticas e nativas usadas como fonte energética no polo gesseiro do Araripe- PE. 2009. 65 f. Dissertação (Mestrado em Ciências Florestais). Universidade Federal Rural do Pernambuco, Recife.

BATISTA, J. L. F.; COUTO, H. T. Z. O "Estéreo". METRVM, n.2, 2002.

BRASIL. Ministério de Minas e Energia. Balanço energético nacional. Brasília: MME, 2017.

BRITO, J. O. O uso energético da madeira. Estudos Avançados, v. 21, n. 59, 2007.

CAMPOS, J. C. C.; LEITE, H. G. Mensuração Florestal: perguntas e respostas. 4 ed. atual. ampl. Viçosa, MG: Ed. UFV, 2013, 605p.

LIMA, E. A. Alternativa para estimar o preço da madeira para energia. Colombo: Embrapa, 2010. 3p. (Embrapa florestas. Comunicado Técnico, 260).

LISBOA, G. S.; DIAS, A. N.; VALERIO, F. A.; SILVESTRE, R. Comparação do fator de empilhamento sob diferentes condições para madeira de Eucalyptus grandis. Ambiência, v. 5, n. 1. 2009.

MACHADO, S. A.; FIGUEIREDO FILHO, A. Dendrometria. 2. ed. Guarapuava: UNICENTRO, 2009. 316p.

NASCIMENTO, M. D.; BIAGGIONI, M. A. M. Avaliação energética do uso de lenha e cavaco de madeira para produção de energia em Agroindústria Seropédica. Revista Energia na Agricultura, Botucatu, v. 25, n. 3, p. 104-107, 2010. 
SANTOS, W. S.; HENRIQUES, I. G. N.; SANTOS, W. S.; RAMOS, G. G.; VASCONCELOS, G. S.; VASCONCELO, A. D. M. Análise florísticafitossociologica e potencial madeireiro em área de caatinga submetida a manejo florestal. Agropecuária Científica do Semiárido (ACSA), Patos, v. 13, n. 3, p. 203-211, 2017.

SCOLFORO, J. R. et al. Amostragem e coleta de dados para equações de volume, peso de matéria seca, tanino, cortiça, óleo, sistema radicular, conversão de volume e carbono. In: SCOLFORO, J. R.; OLIVEIRA, A. D.; ACERBI JÚNIOR, F. W. (Ed.). Inventário Florestal de Minas Gerais - Equações de Volume, Peso de matéria seca e carbono para diferentes fisionomias da flora nativa. Lavras: UFLA, 2008. cap. 1, p.1-65.

SCOLFORO, J. R. S.; PÉREZ, J. F. M.; MELLO, J. M.; OLIVEIRA, A. D.; CAMOLESI, J. F.; BORGES, L. F. R.; ACERBI JÚNIOR, F. Estimativas de volume, peso seco, peso de óleo e quantidade de moirões para a candeia (Eremanthus erythropappus (DC.) MacLeish). Cerne, Lavras, v. 10, n.1, p. 87-102, 2004.

SOARES, C. P. B. RIBEIRO, J. C. NASCIMENTO FILHO, M. B. RIBEIRO, J. C. L. Determinação de fatores de empilhamento através de fotografias digitais. Revista Árvore, Viçosa, v. 27, n. 4, p. 473-479, 2003. 